\title{
Improved Satellite Scheduling Algorithm for Moving Target
}

\author{
Guanlin Mei ${ }^{1}$ \\ National Digital Switching System Engineering and Technological R\&D Center \\ Zhengzhou,450001, China \\ E-mail: hy_meiling163.com

\section{Xiaomin $\operatorname{Ran}^{2}$} \\ National Digital Switching System Engineering and Technological R\&D Center \\ Zhengzhou,450001, China \\ E-mail: $1006773667 @ q q$.com
}

\section{Deliang Fang ${ }^{3}$}

National Digital Switching System Engineering and Technological R\&D Center Zhengzhou,450001 China

E-mail: 1153496660@qq.com

\section{Ce Zhang ${ }^{4}$}

National Digital Switching System Engineering and Technological R\&D Center Zhengzhou,450001, China

E-mail: cezhangefoxmail.com

In order to solve the disturbance to the surveillance strategy caused by uncertainty from the moving target and the satellite inherent defects, an algorithm, which can adaptively adjust the weights based on the real-time distribution probability of the target in task area in combination with the detection probability and Kullback-Leibler (KL) discriminant is proposed. KL discriminant is introduced to measure the uncertainty. The detection probability which can overcome the defect that the area has larger distribution probability will be ignored by the method of KL discriminant, then the satellite's ability to find target will be improved. For the purpose of analyzing and testing the algorithm, a simulation experiment introducing a variety of algorithms was carried out and the computational results verified the algorithm's validity.

ISCC 2015

18-19, December 2015

Guangzhou, China

\footnotetext{
${ }^{1}$ Speaker

${ }^{2}$ This study is supported by National Science and Technology Major Project (2014zx03006003)

${ }^{3}$ Corresponding Author
} 


\section{Introduction}

With continuous development of the satellite technology, the satellite plays an increasingly important role in terms of environment monitoring, traffic control, fishery surveillance and accident rescue, especially in recent years, the marine and air disaster occurred frequently, the satellite surveillance technology featuring wide coverage area, long duration and few restriction by geographical conditions and airspace borders has become an important technical support means [1]; therefore, the exploration and research of satellite surveillance technology will have a broad application prospect and significant value.

At present, some groups studied the scheduling algorithm for the point target [1-2]; some groups studied the scheduling algorithm for regional targets and achieved good results [3-4]. Yet satellite surveillance technology for moving target is still on the exploratory stage. According to the characteristics of the moving target, Ciyuanzhuo proposed a solution for moving target by converting the moving target to the regional target [5], but the satellite was unable to find the target effectively in the surveillance period by using coverage rate as optimization index, which ignored the uncertainty from moving target. Ranchengxin dynamically built regions that may cover the moving target based on the prior information[6], and built the scheduling model in combination with the detection probability and coverage rate. He improved the satellite's ability of finding the target but the uncertainty from moving target was still a problem. Considering the uncertainty from the moving target and the uncertainty of satellite itself, Paul E. Berry and Carmine Pontecorvo from Defense Science and Technology Organization of Australia (DSTO) used information entropy as the optimization goal for scheduling [7], they have obtained certain effects, but the simulation results illustrated the information entropy model was no better than the probability model on the early stage of satellite scheduling period. A scheduling model combining with information gain and detection probability has been put forward in the doctorate dissertation of Ciyuanzhuo[8], but the combination of two kinds of income indexes was relatively simple and the calculation method of information gains can also be further improved. On the basis of the above research, this paper makes a further research for moving target. A new optimization index using KL divergence to measure information and further optimize the combination of two indicators is put forward.

\section{Basis of the Scheduling Algorithm for Moving Target}

\subsection{Satellite Resources}

According to the type of satellite borne sensors, earth surveillance satellite can be divided into Visible Light, Multi-spectral, SAR, Infrared, Hyper Spectral Hyperspectral and Surface Electromagnetic Detection [9], and the surveillance method and scope are also different. This paper uses the image satellite as an example to carry on the analysis and explanation. Optical image satellite in orbit can image different ground moving targets based on different demands. The image data obtained by the satellite can be recorded on films and memory storage devices or transmitted to the ground station through space links (one part of the aerospace test and control system). Then the ground central station can obtain all sorts of valuable information by processing and interpreting the recycling data. The optical image satellite has the characteristic 
of high resolution, but higher resolution usually brings about narrower imaging width. The image satellite is also greatly influenced by the lighting and the weather conditions.

\subsection{Task Area Division}

The task area should span the entire area where a target could exist in the whole surveillance period, and the task area should be a rectangular area for processing problem more conveniently. According to the geographical location coordinates, the task area can be indicated as $I=\left[\left(L O N_{l}, L A T_{l}\right),\left(L O N_{r}, L A T_{r}\right)\right]$ based on longitude and latitude of the upper left and lower right corner, which will be conducive to our research of scheduling algorithm but not affect the results.

In order to establish quantitative indexes and facilitate the analysis of the problem, the task area should be meshed based on longitude and latitude while the grids should be numbered in order $1,2,3, \ldots N$. The partition of the grids must be careful because the size of a grid should not be too big or too small. The size of a grid too large will lead to low location accuracy but too small will lead to a huge number of grids which will cause expensive computational costs. On the basis of the prior information, the probability of a grid being occupied by a target can be defined as $p_{i}, i=1,2,3, \ldots N$.

\subsection{Moving Target Model}

Using Gaussian motion model as put forward in the paper of Paul E. Berry[7], assume that the target location at time $t_{n}$ be $\left(x_{n}, y_{n}\right)$ and at time $t_{n-1}$ be $\left(x_{n-1}, y_{n-1}\right)$, then the $x$ and $y$ components of the position are independent. Let $m_{n}$ be the number of time steps $\Delta t$ from time $t_{n}$ to time $t_{n-1}$. Assume that the target has constant speed in a time step $\Delta t$, and the acceleration from time $t_{n}$ to time $t_{n-1}$ is white Gaussian sequence $\varepsilon_{n}$, with the same variance

$\xi$. Acceleration is constant over a time step $\Delta t$. Then the speed $\dot{x}_{n}$ and location $x_{n}$ at time $t_{n}$ to the $x$ direction as follows (those for $y$ direction are similar):

$$
\begin{gathered}
\dot{x}_{n}=x_{n-1}^{\cdot}+m_{n} \Delta t \varepsilon_{n} \\
x_{n}=x_{n-1}+m_{n} \Delta t x_{n-1}+\frac{1}{2}\left(m_{n} \Delta t\right)^{2} \varepsilon_{n}
\end{gathered}
$$

So the target transition probability can be denoted by $p\left\{\left(x_{n}, y_{n}\right) \mid\left(x_{n-1}, y_{n-1}\right)\right\}$ and is known to be bivariate Gaussian with $x_{n}$ and $y_{n}$ having mean

$$
\begin{aligned}
& \mu_{x}=x_{n-1}+m_{n} \Delta t x_{n-1}^{\cdot} \\
& \mu_{y}=y_{n-1}+m_{n} \Delta t y_{n-1}^{\cdot}
\end{aligned}
$$

Then transition probability from location $\left(x_{n-1}, y_{n-1}\right)$ to location $\left(x_{n}, y_{n}\right)$ can be expressed as:

$$
p\left\{\left(x_{n}, y_{n}\right) \mid\left(x_{n-1}, y_{n-1}\right)\right\}=\frac{1}{2 \pi \sigma^{2}} \exp \left(\frac{-1}{2 \sigma^{2}} \Delta l^{2}\right)
$$

Here, The variances value $\Delta l=\sqrt{\left(x_{n}-\mu_{x}\right)^{2}+\left(y_{n}-\mu_{y}\right)^{2}}$ and $\sigma^{2}=\frac{4 \mathrm{~m}_{n}^{3}-m_{n}}{12} \xi \Delta t^{4}$. 


\section{Scheduling Algorithm based on Information Measurement and Detection Probability}

The surveillance problem for moving target can be described as: there are a given task area $I$ and satellite resources. Assume the time window satellites obtained at discrete instants of time $t_{1}, t_{2, .} \ldots t_{n}$ be expressed as [tStartntEndn] at the time $t_{n}$. For any discrete time $t_{n}$, according to the results of surveillance at time $t_{n-1}$ (based on the prior information acquired at time $t_{1}$ ), forecast the target's location at time $t_{n}$, and update the distribution probability of the area, then select the optimal swath, namely the actual surveillance area, based on the constraint conditions such as distribution probability, satellite position and current sensor's ability, and the optimization indexes, finally transfer the data obtained at time $t_{n}$ to the ground station. When the next satellite has a time window, repeat the process until the end of scheduling task.

Satellite scheduling methods have unreal-time scheduling model and real-time scheduling model, but the scheduling algorithm should have high instantaneity for moving target; therefore, this paper uses the real-time scheduling model.

\subsection{Satellite Surveillance Action Set}

The satellite time windows that satellite access the task area at discrete instants of time $t_{1}, t_{2, .} \ldots t_{n}$ are usually not too long. The space-borne sensors can continue boot for task area surveillance within the time window. The task area can't be covered by one surveillance because the satellite width is limited; thus the task area should be divided into swaths based on the image width of satellite, track of sub-satellite points and satellite side-sway angle, and the satellite surveillance action set can be built. Bring in the swath division method based on the position of satellite relative to the task area [10-11]. Assume that field of view (FOV) of satellite $i$ be $\varphi_{i} \in \mathbb{R}$, the time window of satellite access to the task area at $j$ times be $\operatorname{win}_{i j}$, and sidesway angle be $\psi_{i j l}$, then the surveillance swath can be described as $S_{i j l} \in S=\operatorname{Swath}\left(\operatorname{win}_{i j}, \psi_{i j l}, \varphi_{i}\right)$, and the number of grids the swath contains is $G$. The swaths are shown in Fig. 1:

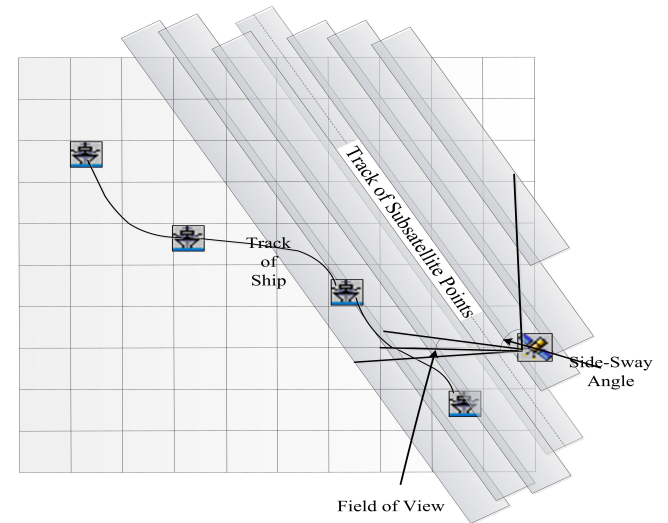

Figure 1: Swaths Division of Target Area

When the satellite accesses the target area, the satellite's accession time window and FOV are constant value and the satellite surveillance action set is controlled by the side-sway angle; so the surveillance action set of satellite $i$ access to the task area at $j$ times is:

$$
A_{i j}=\left\{S_{i j l} \mid \psi_{i j l}=\Delta \psi_{i} \cdot l \in\left[-\psi_{i j l}^{\max }, \psi_{i j l}^{\max }\right], l \in \mathbb{Z}\right\}
$$


$\left[-\psi_{i j l}^{\max }, \psi_{i j l}^{\max }\right]$ denotes the range of side-sway angle.

\subsection{Satellite Scheduling Optimization Index}

The detection probability is a kind of traditional income index for moving target, but it doesn't consider uncertainties from defects of the space-borne sensor, target motion characteristic and complex environment. TheI information income index considers the uncertainties in the process of scheduling, but may ignore the essential requirement of finding the target. Ciyuanzhuo proposed a optimizing index combined with the information income and the detection probability income [8]. In this paper, on the basis of Ciyuanzhuo, a new satellite scheduling optimization index that introduces the KL divergence and optimizes the combination of information income and detection probability income is hereby proposed.

Suppose that the prior distribution probability when the satellite accesses the task area be $\hat{p}_{i}, i=1,2,3, \ldots N$, and $\sum_{i=1}^{N=1} \hat{p}_{i}=1$. The information gain can be calculated based on the posteriori distribution probability, but the posteriori distribution probability of target can't be known at decision-making stage. Hence assume the implementing surveillance be $S_{i j l}$, which denotes the swath corresponding to side-sway angle $l$ of satellite $i$, and obtain the surveillance result $z$, then the distribution probability for a target updated by bayes' rule is shown as follows:

$$
p_{i}=\frac{p(z \mid i) \cdot \hat{p}_{i}}{p(z)}
$$

Here, $p(z \mid i)$ is defined by the satellite sensor model which is shown as follow:

When target is in grid $i, \quad p(z \mid i)$ is

$$
p(z \mid i)=\left\{\begin{array}{c}
p_{d}\left(1-p_{f}\right)^{G-1} z=1 \\
\left(1-p_{d}\right)\left(1-p_{f}\right)^{G-1} z=0
\end{array}\right.
$$

Here, $p_{d}$ is the satellite's detection probability, $p_{f}$ is the false alarm probability.

When the target is not in grid $i$, but in the swath satellite selected, $p(z \mid i)$ is

$$
p(z \mid i)=\left\{\begin{array}{c}
p_{f}\left(1-p_{d}\right)\left(1-p_{f}\right)^{G-1} \quad z=1 \\
\left(1-p_{f}\right)^{G} \quad z=0
\end{array}\right.
$$

When the target is not in the swath satellite selected, $p(z \mid i)$ is

$$
p(z \mid i)=p_{f}\left(1-p_{f}\right)^{G-1} \quad z=1
$$

In practice, it's impossible to get continuous distribution, then the KL divergence is:

$$
D=\sum_{i=1}^{N} p_{i} \log \left(\frac{p_{i}}{\hat{p}_{i}}\right)
$$

As the surveillance is not really implemented, the actual result of surveillance can't be known; therefore, considering all possible results, the information income is:

$$
f_{I}(j)=E_{z}\{D\}=\sum_{z=0}^{1} \sum_{j=1}^{N} p(z) p_{j} \log \left(\frac{p_{j}}{\hat{p}_{j}}\right)
$$

For the detection probability, assume the swath $S_{i j l}$ contain grid set $G_{S_{i j t}}=\left\{g_{1}, g_{2,}, \ldots, g_{k}\right\}$. Suppose that the number of grids in the swath be $N_{g}$, then the then detection probability income is: 


$$
f_{D}(j)=\sum_{k=1}^{N_{g}} \hat{p}_{g_{k}} p_{d}
$$

$p_{d}$ denotes the detection probability established by the capability of satellite itself such as image resolution.

The information income and the detection probability income have different units, so they need to be normalized. The sum of all possible information incomes and the detection probability incomes obtained by observing all swaths in satellite surveillance action set are expressed as:

$$
\begin{gathered}
F_{I}=\sum_{S_{i j} \in A_{i j}} f_{I}(j) \\
F_{D}=\sum_{S_{i j l} \in A_{i j}} f_{D}(j)
\end{gathered}
$$

The algorithm optimization index can be expressed as:

$$
\max _{S_{i j i} \in A_{i j}} F=\max _{S_{i j l} \in A_{i j}}\left(\omega_{1} \frac{f_{I}(j)}{F_{I}}+\omega_{2} \frac{f_{D}(j)}{F_{D}}\right)
$$

Here, $\omega_{1}$ and $\omega_{2}$ denote the preference weight. Both information income and detection probability income have their advantages and disadvantages; then the uncertainty from moving target makes the information income to play a vital role in the scheduling algorithm. However, satellite may select the grid that target distribution probability is lower for reduce uncertainty when using the information income as optimization index, resulting in unable to find target effectively; therefore this paper sets dynamic weights to overcome the problem, let $\omega_{2}=\underset{S_{i j t} \in A_{i j}}{\max }\left(\sum_{k=1}^{N g} \hat{p_{g_{k}}}\right) \quad$ which is the maximum value of sum of target distribution probability in the surveillance swath, and $\omega_{1}+\omega_{2}=1$, then $\omega_{1}=1-\max _{S_{j i t} \epsilon A_{i j}}\left(\sum_{k=1}^{N g} \hat{p_{g_{k}}}\right)$. The weights dynamically change with the distribution probability. In this way, the information income takes the dominant position when the sum of target distribution probability in the surveillance swath is small, and yet the detection probability takes the dominant position when the sum of target distribution probability in the surveillance swath is big. The optimization index introduced above avoids the deficiency of the information income.

Based on the description above, the scheduling algorithm can be described as:

Step 1: calculate the surveillance action set $A_{i j}$ based on the surveillance window win $_{i j}$ by calculating the satellite access time and the corresponding conditions.

Step 2: calculate the target distribution probability $\hat{p}_{i}, i=1,2,3, \ldots N$, when the satellite accesses the task area based on target transition probability (calculate the target distribution probability based on the prior conditions when the satellite accesses the task region first time).

Step 3: compute the information income and the detection probability income by traversing the surveillance action set.

Step 4: comprehensive index is used to select the optimal surveillance swath as follows:

$$
S_{i j l}=\max _{S_{i j l} \in A_{i j}} F=\max _{S_{i j l} \in A_{i j}}\left(\omega_{1} \frac{f_{I}(j)}{F_{I}}+\omega_{2} \frac{f_{D}(j)}{F_{D}}\right)
$$


Step 5: apply the Bayes' rule to update the target distribution probability. Schedulee stop if the surveillance task is at the end; otherwise go to Step 1.

It is worth noting that there is a certain delay before the satellite receives the surveillance results from last step, so the surveillance results acquired at the last step can't be used for establishing the scheduling strategy when the satellite accesses the task area. If the satellite can't obtain the result timely, we need to use earlier time feedback result as the prior condition.

\section{Simulations}

In order to facilitate the simulation, assume the problem doesn't take the feedback delay into consideration, and it doesn't affect the validity analysis of scheduling algorithm. Suppose the task area be rectangle and the task area is discretized into numbered grids. There is a ship running in the task area at any curve at speed of about 30 knots, namely 55.56 . The route of target is shown in Fig. 3:

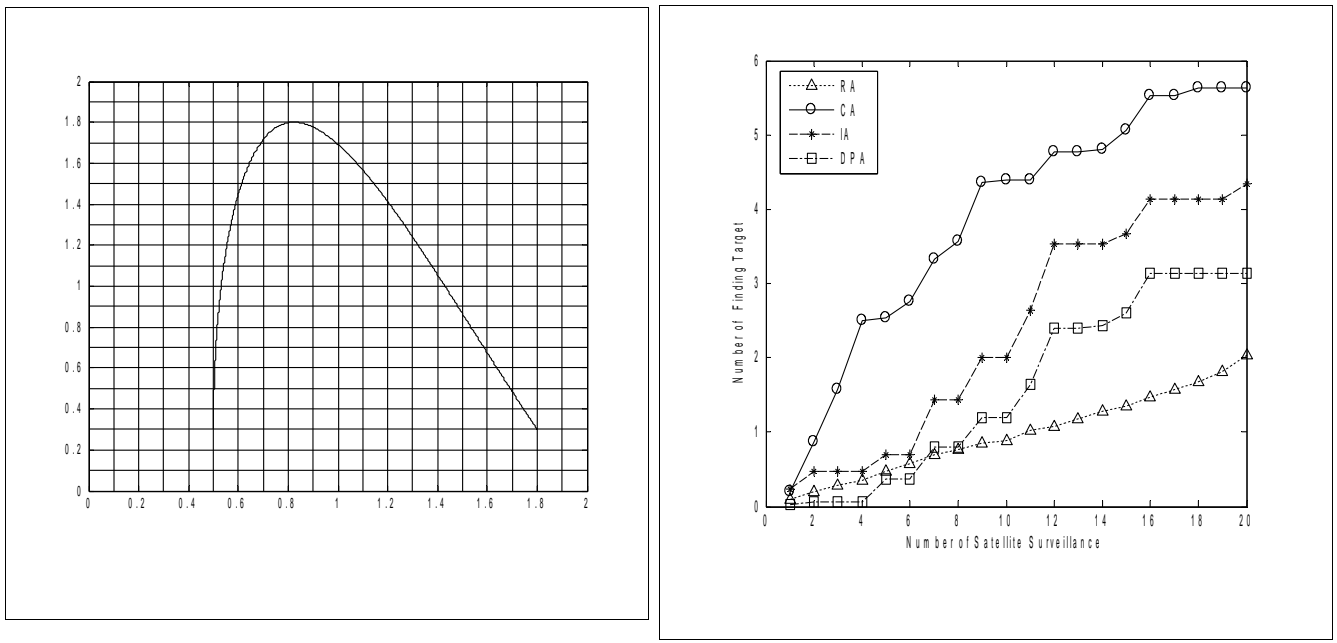

Figure 2: Target Motion Trajectory Figure 3: Algorithm Performance Comparison

In order to verify the validity of the comprehensive algorithm, this paper makes a comparison of random selection algorithm(RA), detection probability algorithm (DPA), information algorithm(IA) and the comprehensive algorithm(CA). The random selection algorithm randomly selects one swath in the satellite surveillance action set every time satellite accesses the task area; the detection probability algorithm selects swath with the objective of maximizing the sum of detection probability of all grids in the surveillance swath as income index; the information algorithm uses information gain as the optimization index to select swath.

Assuming that the satellite's detection probability be $p_{d}=0.9$, the false alarm probability is $p_{f}=0.03$. In order to eliminate the effects of random initial conditions, the results of the average cumulative number of finding by calculating 100 groups of scheduling experiments which have random prior to the distribution probability are shown in Fig. 4:

Abscissa denotes the number of time windows satellite obtained and ordinate denotes cumulative number of finding the target. the The detection probability model in the initial stage is slightly worse than random model, and the comprehensive algorithm is more advanced than other two kinds of algorithms can be seen from the results in Fig. 3, which means the algorithm 
effectively improves the performance of satellite scheduling algorithm. It is important to note that this algorithm adds to the computation cost in comparison to the other two algorithms, but for the efficient improvment of finding target, this algorithm is still have advantages.

\section{Conclusion}

This paper proposes a new satellite sensor scheduling algorithm for moving target. From the above computer simulation examples, we can see that:

1) the algorithm puts forward the ability to find the moving target. The average cumulative number of finding target increases from 2 to more than 5 within 20 times.

2) the computational complexity of the algorithm has been reduced by using KL divergence instead expected information entropy, and KL divergence can be extended to arbitrary probability space; so KL divergence has a more general significance.

3) this algorithm integrates the advantages of information income and detection probability income, and effectively improves the ability of finding target on the initial stage.

\section{References}

[1] J. Frank, A. Jonsson, R. Morris, et al. Planning and Scheduling for Fleets of Earth Observing Satellites $[\mathrm{C}]$. Proceeding of the 6th International Symposium on Artificial Intelligence, Robotics, Automation and Space. Montreal.pp,1-12, 2003.

[2] A. Globus, J. Crawford, J. Lohn, et al. Earth Observing Fleets Using Evolutionary Algorithms: Problem Description and Approach[C].Proceedings of the 3rd International NASA Workshop on Planning and Scheduling for Space, America: NASA.pp,27-29(2003)

[3] R. Cohen. Automated Spacecraft Scheduling-the Aster Example[R]. America, Jet propulsion Laboratory,2002.

[4] D. Z. Zhang, L. Guo, Q. Wang, et al., An improved single - orbit Scheduling Method for Agile Imaging Satellite forward Area Target [J]. Journal of Geomatics and Information Science of Wuhan University, 2014, 33 (8) 6:901-905 .

[5] Y. Z. Ci, B. C. Bai, Q. M. Ruan, et al. Moving Target Reconnaissance by Multi- $\quad$ Satellite: A strategy Based on Latent Area[J]. Chinese Journal of Sensors and Actuators, 2008, 21 (6) : 10151019.

[6] C. X. Ran, H. L. Wang, Gangyao. Xiong, et al. Research on Mission-Planning of Ocean Moving Targets Imaging Reconnaissance Based on Improved Genetic Algorithm[J]. Journal of Astronautics, 2010, $31(2): 457-465$.

[7] P. E. Berry, C. Pontecorvo, D. A. B. Fogg. Optimal Search, Location and Tracking of Surface Maritime Targets by a Constellation of Surveillance Satellites[R].Australia: Intelligence, Surveillance and Reconnaissance Division Information Sciences Laboratory, 2003.

[8] Y. Z. Ci. Multi-Satellite Mission Planning for Moving Target Search[D]. Changsha: National University of Defense Technology, 2008.

[9] W. Jiang, H. C. Hao, Y. J. Li. Review of task scheduling research for the Earth observing satellites[J]. Systems Engineering and Electronics, 2013, 35 (9) : 1878-1885

[10] Y. F. Xu. Joint Scheduling for Space-based Maritime Moving Targets Surveillance[D]. Changsha: National University of Defense Technology, 2011.

[11] Y. H. Guo. The Study on Key technologies Multiple Types of Earth Observing satellites United Scheduling[D]. Changsha: National University of Defense Technology, 2009. 